\title{
Urgência Oftalmológica: Corpo estranho ocular ainda como principal causa
}

\author{
Ophthalmologic Emergency: Foreign bodies still are the principal cause
}

\author{
Ângelo Auguusto da Silva Araújo ${ }^{1}$ \\ Davison Vieira Almeida ${ }^{2}$ \\ Vitalina Martins de Araújo ${ }^{2}$ \\ Max Rollemberg' Góes ${ }^{3}$
}

\section{RESUMO}

Objetivo: Os distúrbios traumáticos constituem a grande maioria das urgências oftalmológicas. O conhecimento prévio dos fatores de risco epidemiológicos possibilita trabalhos de medicina preventiva, dentre outros. Este estudo avalia os motivos que conduzem os pacientes a procurarem um serviço de emergência oftalmológica. Métodos: Foram cadastrados, em um "software" exclusivo, os prontuários dos pacientes atendidos entre os dias 01/01/2000 e 20/10/2000 no Hospital Governador João Alves Filho, Aracaju / SE - Brasil. Selecionaram-se 859 pacientes, de acordo com critérios previamente estabelecidos, analisando as seguintes variáveis: sexo, idade, dia da semana, mês, diagnóstico e conduta médica adotada. Resultados: O sexo masculino representou $72 \%$ no total de atendimentos; o diagnóstico de trauma foi encontrado em $52 \%$ dos casos, sendo que 59,5\% correspondiam ao corpo estranho ocular; as consultas nos dias úteis superaram em 62,5\% as dos dias não-úteis. Conclusão: O sexo masculino demonstrou maior vulnerabilidade ao trauma, visto que a faixa etária mais acometida foi a economicamente ativa. A etiologia mais freqüente foi corpo estranho ocular e o atendimento em dias úteis foi maior do que nos dias não-úteis.

Descritores: Serviços médicos de emergência; Serviço hospitalar de emergência; Corpos estranhos no olho/epidemiologia; Traumatismos oculares/epidemiologia; Acidentes de trabalho

\section{INTRODUÇ̃̃̃O}

A população, de uma forma geral, está exposta a diversos fatores de risco que podem levar as pessoas a procurarem atendimento médico de urgência, e dentre eles, estão os que podem acometer os olhos. Os estudos analisados $^{(1-4)}$ demonstram que as situações que levam os pacientes a procurarem um serviço de oftalmologia de urgência são diversas, abrangendo desde alterações visuais que podem causar baixa visual súbita e permanente, a desordens que causam desconforto visual.

De certo, a quantidade de pacientes atendidos no setor de urgência/ emergência em oftalmologia varia em torno de $7 \%^{(1)}$ do total das consultas de um hospital geral. Foi demonstrado ainda ${ }^{(1-4)}$, que a prevalência dos atendimentos de pacientes do sexo masculino é relevante quando comparada a do sexo feminino. Do mesmo modo, a população de idade economicamente ativa foi mais acometida e, dentre estas, destacaram-se os traumas.

Desse modo, o presente estudo tem como finalidade o conhecimento e a análise dos diversos fatores de riscos epidemiológicos, como também de patologias que fazem parte das mais variadas situações de emergências 
oftalmológicas. Dentre suas várias possibilidades etiológicas, enfatizamos neste estudo o trauma provocado pela entrada e fixação de um corpo estranho ao nível da região ocular.

\section{MÉTODOS}

Foi realizado o levantamento da população a ser estudada dos pacientes atendidos no Hospital Governador João Alves Filho (HGJAF), Aracaju / SE - Brasil, no período compreendido entre 01/01/2000 a 20/10/2000. A partir disso, calculou-se o número de pacientes cadastrados necessário para obtenção de uma amostragem estatisticamente válida ${ }^{(5)}$.

Em uma amostra piloto de 30 pacientes foi demonstrado que a Probabilidade da ocorrência de uma afecção traumática (P) foi de $45 \%$ e a Probabilidade da ocorrência de uma patologia não traumática (Q) foi de $65 \%$ (que corresponde a $100 \%$-P); diante desses dados, o número de pacientes necessários para um estudo representativo foi calculado e definido como índice de erro amostral neste estudo 0,03 (3\%).

Dessa forma, o número de pacientes necessário para obtenção de uma amostra estatisticamente válida foi de 808 .

A etapa posterior foi seguida da construção de software adequado para o cadastramento e análise das variáveis: sexo, idade, data de admissão hospitalar, classificação diagnóstica e conduta médica. Entre 01/04/2000 a 31/07/2000, foram cadastrados 1224 pacientes e excluídos 365 , ficando 859 pacientes, pelos seguintes motivos:

- Alguns pacientes evadiram-se do local sem aguardar atendimento.

- Vários prontuários não tinham sido preenchidos pelos médicos com qualquer informação.

- Vários pacientes eram portadores de desordens avaliadas em nível ambulatorial, não necessitando de atendimento de urgência/emergência.

- Alguns pacientes foram erroneamente encaminhados ao setor de oftalmologia.

- Alguns pacientes foram atendidos por necessidade de revisão de procedimentos executados anteriormente à admissão.

A leitura dos prontuários e o cadastramento dos dados foram realizados por uma única pessoa. A seguir foi utilizado de metodologia estatística, o teste Qui Quadrado $\left(\chi^{2}\right)$, para verificar o Grau de independência dessas variáveis.

\section{RESULTADOS}

O presente estudo se baseou na análise dos prontuários de 859 pacientes do referido período, perfazendo uma amostragem estatisticamente válida dos 3041 pacientes atendidos no setor de oftalmologia no período de 01/01/2000 a 20/10/2000.

Assim, considerando os 859 pacientes cadastrados, a fre- quiência de pacientes encontrados foi de acordo com os seguintes resultados:

\section{Em relação a variável Dia da Semana: (Gráfico 1)}

A freqüência do total de pacientes admitidos no setor de urgência/emergência oftalmológica depende do Dia da Semana que os pacientes foram admitidos. $\left(\chi_{\text {Calc }}^{2}=42,94\right.$, sendo o $\left.\chi_{\mathrm{Tab}}^{2}=12,6\right)$

\section{Em relação a variável Sexo: (Gráfico 2)}

A frequiência do total de pacientes admitidos no setor de urgência/ emergência oftalmológica depende do sexo dos pacientes admitidos. $\left(\chi_{\text {Calc }}^{2}=165,45\right.$, sendo o $\left.\chi_{\text {Tab }}^{2}=3,84\right)$

Em relação a variável Grupo de Idade: (Gráfico 3)

A frequiência do total de pacientes admitidos no setor de urgência/ emergência oftalmológica depende do grupo de idade em que os pacientes admitidos se encontram. $\left(\chi_{\text {Calc }}^{2}=260,42\right.$, sendo o $\chi_{\text {Tab }}^{2}=9,49$ )

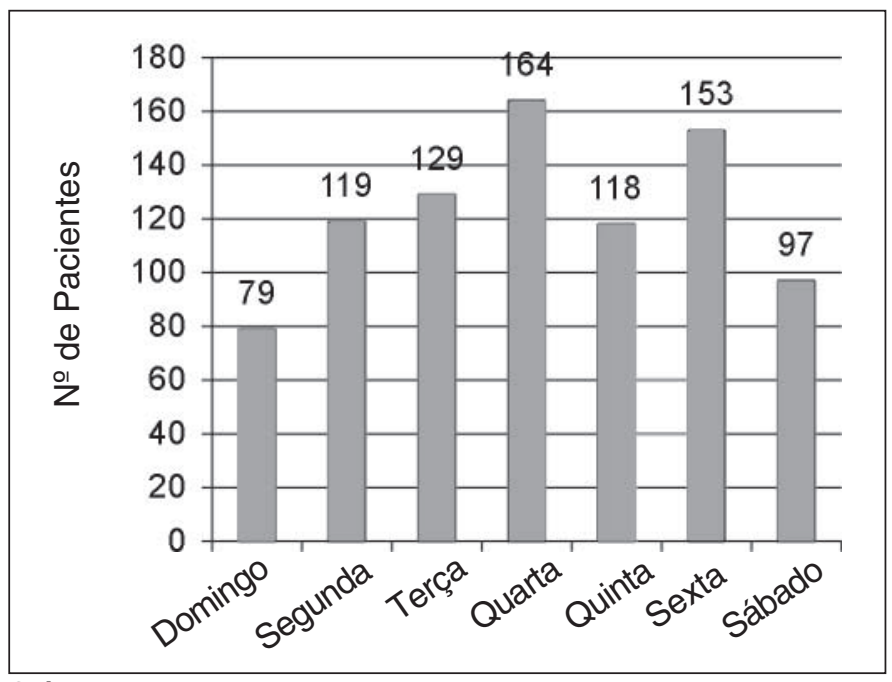

Gráfico 1 - № de pacientes $X$ dia da semana

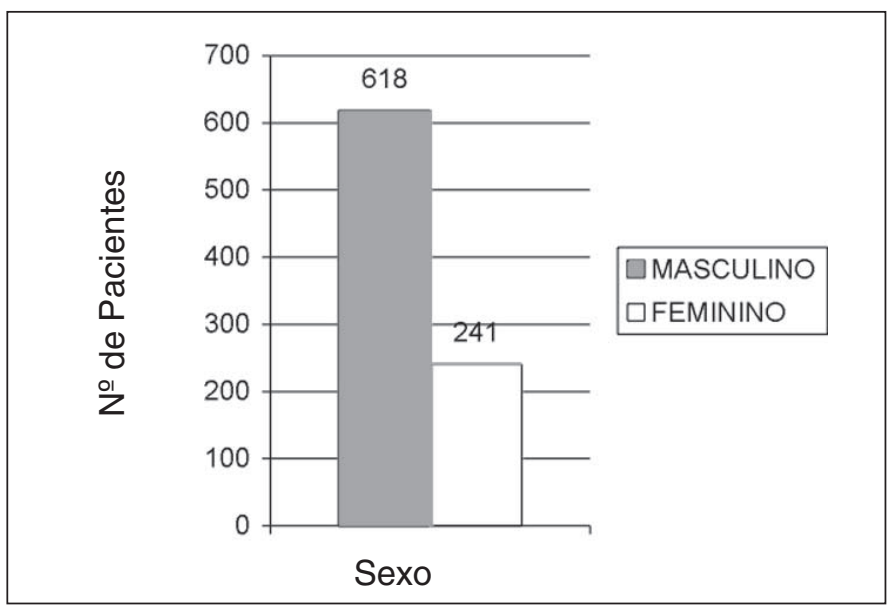

Gráfico 2 - № de pacientes $X$ sexo 


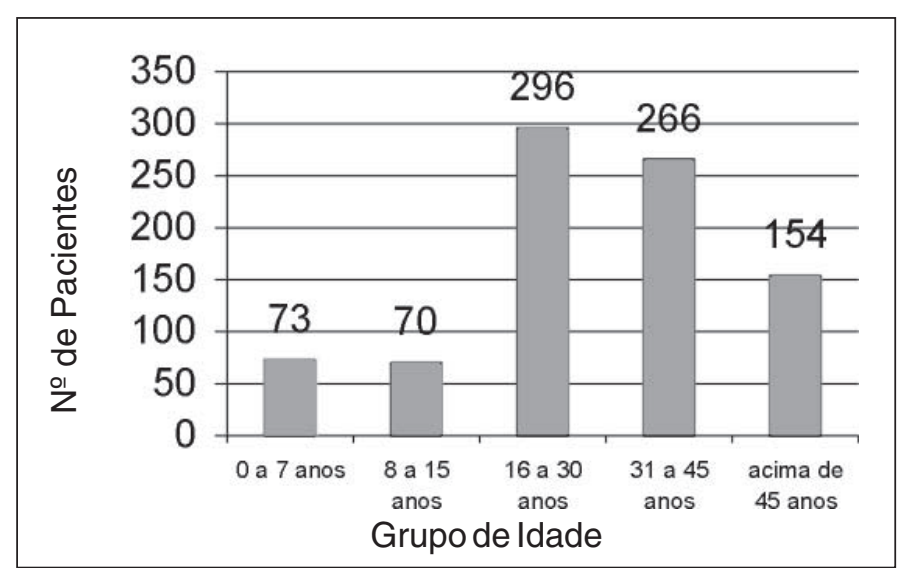

Gráfico 3 - Nº de pacientes $X$ grupo idade

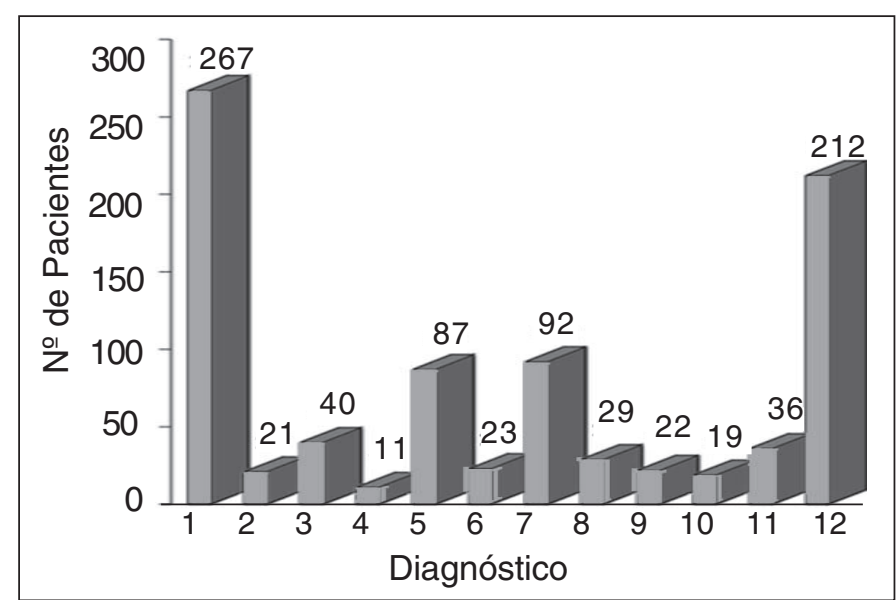

Gráfico 4 - $\mathrm{N}^{\circ}$ de pacientes $X$ diagnóstico

\section{Em relação a variável Diagnóstico: (Gráfico 4)}

1 - Corpo estranho ocular (incluindo córnea, conjuntiva e regiões intra-oculares).

2 - Trauma do tipo perfuro-cortante.

3 - Trauma do tipo contuso.

4 - Queimaduras oculares e em anexos (pálpebra superior e inferior).

5 - Traumas não especificados pela anamnese.

6 - Acidentes envolvendo contato ocular de produtos químicos.

7 - Conjuntivites não traumáticas, abrangendo as infecciosas e as alérgicas.

8 - Úlcera de córnea ou ceratites.

9- Glaucoma agudo.

10 - Celulites peri-orbitárias.

11 - Hordéolo.

12 - Outros diagnósticos.

A freqüência do total de pacientes admitidos no setor de urgência/ emergência oftalmológica depende do diagnóstico dos pacientes admitidos. $\left(\chi_{\text {Calc }}^{2}=1067,97, \chi_{\text {Tab }}^{2}=19,7\right)$

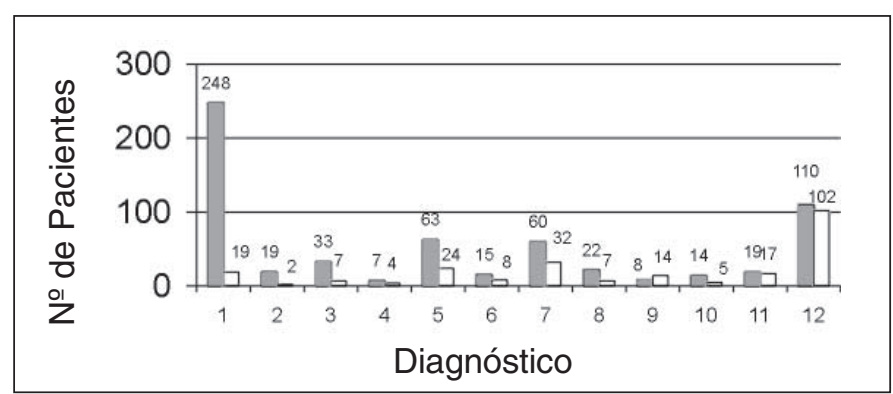

Gráfico 5 - № de pacientes $X$ diagnóstico $X$ sexo

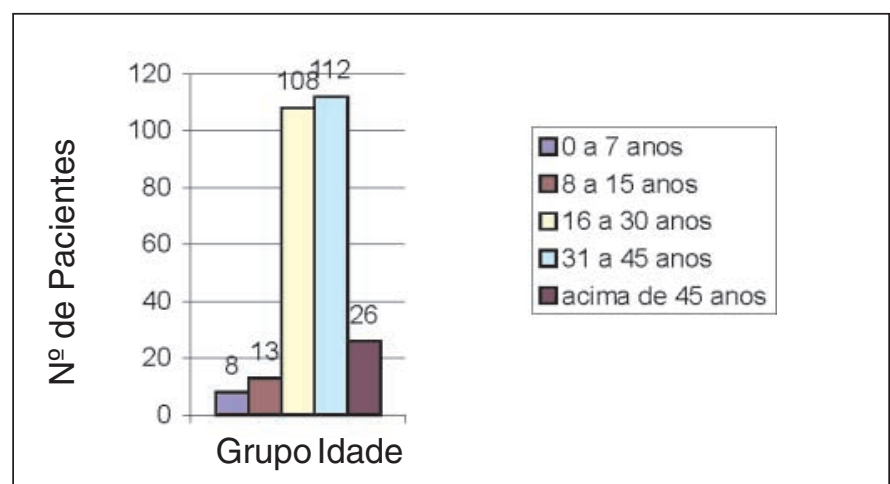

Gráfico 6 - № de pacientes $X$ diagnóstico de corpo estranho ocular $X$ grupo idade

Em relação ao cruzamento das variáveis Diagnóstico e Sexo: (Gráfico 5)

A freqüência do total de pacientes admitidos no setor de urgência/ emergência oftalmológica em relação ao total de diagnósticos é dependente do sexo dos pacientes admitidos. $\left(\chi_{\text {Calc }}^{2}=129,59\right.$, sendo o $\left.\chi_{\text {Tab }}^{2}=19,67\right)$

Em relação à variável Grupo de Idade considerando o Diagnóstico de Corpo Estranho Ocular (1): (Gráfico 6)

A frequiência do total de pacientes admitidos no setor de urgência/ emergência oftalmológica em relação ao diagnóstico de corpo estranho ocular é dependente do grupo de idade em que os pacientes admitidos se encontram. $\left(\chi_{\text {Calc }}^{2}=203,35\right.$, sendo o $\chi_{\text {Tab }}^{2}=9,49$ )

Em relação à variável Dia da Semana considerando o Diagnóstico Corpo Estranho Ocular (1): (Gráfico 7)

A freqüência do total de pacientes admitidos no setor de urgência/ emergência oftalmológica em relação ao diagnóstico de corpo estranho ocular é dependente do dia da semana em que os pacientes foram admitidos. $\left(\chi_{\text {Calc }}^{2}=16,95\right.$, sendo o $\left.\chi_{\text {Tab }}^{2}=12,59\right)$

Em relação ao cruzamento das variáveis Diagnóstico e Grupo de Idade: (Gráfico 8)

A freqüência do total de pacientes admitidos no setor de urgência/ emergência oftalmológica em relação total de diagnósticos é dependente do grupo de idade em que os pacientes admitidos se encontram. $\left(\chi_{\text {Calc }}^{2}=199,4\right.$, sendo o $\left.\chi_{\text {Tab }}^{2}=60,48\right)$ 


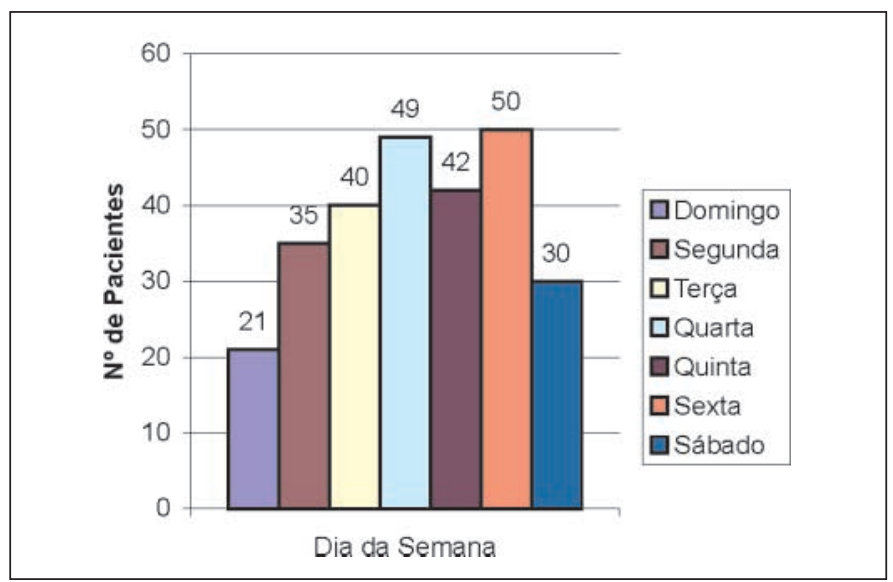

Gráfico 7 - $\mathrm{N}^{\circ}$ de pacientes $\mathrm{X}$ diagnóstico de corpo estranho ocular $\mathrm{X}$ dia da semana

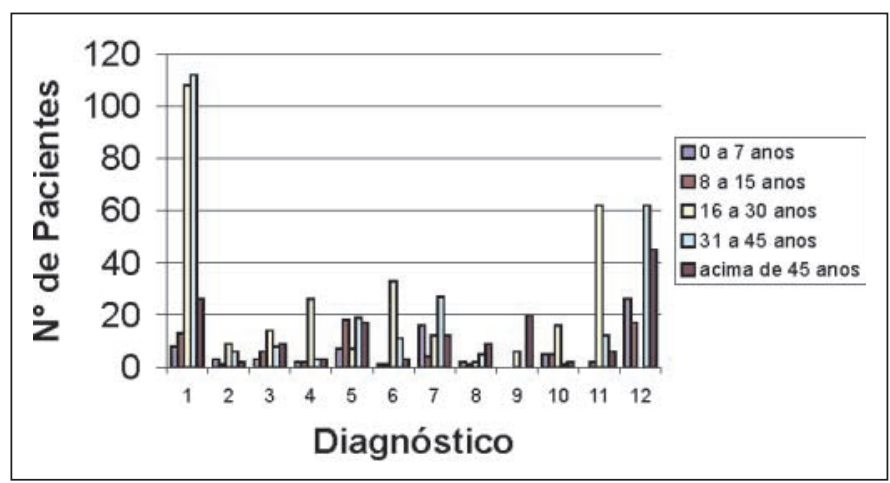

Gráfico 8 - № de pacientes $X$ diagnóstico $X$ grupo idade

\section{DISCUSSÃO}

O setor de urgência/emergência em oftalmologia do HGJAF atende uma baixa quantidade de pacientes em relação ao total geral de consultas realizadas neste hospital. No presente estudo, constatou-se que cerca de $2,2 \%$ do total de admissões eram encaminhados ao setor de oftalmologia, enquanto em outros serviços ${ }^{(1)}$, esse percentual foi de em torno de $7,1 \%$.

Quando se considera apenas o dia da semana, esse estudo demonstrou que no geral o atendimento durante os dias úteis foram mais freqüentes que em relação aos finais de semana. Assim, foram atendidos um total de 683 pacientes de segunda a sexta-feira, contra 176 pacientes admitidos no sábado e no domingo, fixando uma média diária de consultas em torno de aproximadamente 7,8 pacientes nos dias úteis e 4,8 pacientes nos finais de semana, ou seja, em dias úteis atende-se por volta de $62,5 \%$ a mais de que nos dias não-úteis.

Semelhante à literatura, foi encontrado nesse serviço de saúde uma distribuição geral desequilibrada de pacientes por sexo, sendo que $72 \%$ (proporção 3:1) dos atendimentos correspondiam ao sexo masculino, demonstrando uma maior vulnerabilidade deste ao trauma ocular ${ }^{(1-4,6-10)}$. A bibliografia $^{(14)}$ demonstra, em termos gerais, uma variação percentual de admissão para o sexo masculino entre aproximadamente $51 \%$ a $70,9 \%$, e para o sexo feminino cerca de $29,1 \%$ a $48,9 \%$.

Levando-se em conta a faixa etária dos pacientes, verificou-se que os atendimentos ocorriam predominantemente nas faixas etárias compreendidas entre 16 a 45 anos correspondendo a $65,45 \%$ do total de admissões. Quando comparado com os dados bibliográficos ${ }^{(2-4,6,7,10)}$, percebe-se que os dados obtidos são superiores às informações referidas pela literatura (variando de $48,25 \%$ a $58,8 \%$ ). Fazendo o cruzamento das variáveis idade x sexo, observa-se um aumento proporcional do sexo masculino em relação ao sexo feminino $(3,5: 1)$. De acordo com esses resultados, infere-se que se trata de faixa etária da população em idade profissional, a qual está exposta a diversos fatores de risco.

Quando se analisa o diagnóstico, como variável independente, a percentagem de patologias traumáticas situa-se por volta de $52 \%$. Essa freqüência foi encontrada variando em torno de $20 \%$ a $87,3 \%$, nos levantamentos bibliográficos ${ }^{(1-4,6-7)}$. O trauma provocado por corpo estranho ocular (1) predominou como causa etiológica dos traumas, correspondendo a cerca de 59,5\% dos casos, ficando sua frequiência geral em 31,08\% dos diagnósticos. Nos estudos levantados ${ }^{(2,4)}$, os traumas causados por corpo estranho ocular tiveram uma variação de $30,7 \%$ a $47,22 \%$ para as etiologias traumáticas, e de $12,7 \%$ a $26,8 \%$ para o total de diagnósticos. $\mathrm{Na}$ análise por sexo em relação a situações provocadas por traumas, o sexo masculino apresentou uma frequiência total de aproximadamente $85,74 \%$, sendo de $14,25 \%$ o valor referente ao sexo feminino, bastante semelhante aos encontrados em outros estudos ${ }^{(3,6)}$. Quando se considera a relação entre as variáveis sexo x corpo estranho ocular, notamos uma diferença percentual significante entre os sexo masculino $(92,88 \%)$ e feminino $(7,12 \%)$, fixando uma proporção de 13:1 (M/F). A faixa etária também foi bastante relevante nesta afecção, visto que a idade compreendida entre 16 a 45 anos ficou com aproximadamente $82,4 \%$ dos casos. Em relação ao dia da semana $\mathrm{x}$ o diagnóstico de corpo estranho ocular, a média de atendimento diário foi de aproximadamente 2,48 pacientes por dia útil contra aproximadamente 1,42 pacientes nos finais de semana, aumentando a taxa de admissão em 74,64\% nos dias de segunda a sexta em relação a sábado e domingo.

\section{CONCLUSÃO}

As patologias traumáticas constituem o principal motivo que conduz o paciente até um serviço de urgência/emergência oftalmológica. Dentre as enfermidades traumáticas, o trauma causado por corpo estranho na região ocular, representa, ainda, a grande maioria dos atendimentos. O sexo masculino é mais acometido do que o sexo feminino, em uma ordem proporcional de 13:1 (M/F), sendo a faixa etária mais atingida correspondente à idade profissional. Nota-se, com isso, que os 
homens estão mais vulneráveis a esse tipo de acidente, principalmente nos dias úteis. Verificando a população economicamente ativa, percebe-se que a grande maioria ainda é composta pelo sexo masculino, e quando se avalia a relação entre as variáveis estudadas e o trauma por corpo estranho ocular, concluímos que existe uma ligação muito importante relacionada a acidentes ocupacionais.

\section{ABSTRACT}

Purpose: The traumatic diseases constitute the great majority of ophthalmic emergencies. Previous knowledge of epidemiologic risk factors makes possible studies on preventive medicine, among others. This study has the aim to evaluate the reasons which lead to look for an ophthalmologic emergency unit. Methods: The data of patients attended at the "Hospital Governador João Alves Filho", Aracaju, SE - Brazil, between January 1, 2000 and October 20, 2000, were registered according to their charts, using an exclusive software. 859 patients were selected according to previously established criteria and the following variables were analyzed: sex, age, day of the week, month, diagnosis and chosen medical treatment. Results: Males represented $72 \%$ of all cases; trauma diagnosis was established in $52 \%$ of the cases, $59.9 \%$ of them being ocular foreign bodies; cases on working days surpassed by $62.5 \%$ those on weekends. Conclusion: Males showed a greater vulnerability to trauma, since the more affected age range was that of the economically active. The most frequent etiology was ocular foreign body and the number of cases on working days was higher than that on weekends.

Keywords: Emergency medical services; Hospital emergency service; Eye foreign bodies/epidemiology; Eye injuries/ epidemiology; Occupational accidents.

\section{REFERÊNCIAS}

1. Sheldrick JH, Vernon SA, Wilson A, Read SJ. Demand incidence and episode rates of ophthalmic disease in a defined urban population [commented on BJM 1992;305:1501-2]. BJM 1992;305:933-6.

2. Gómez RM. Urgências oftalmológicas. Revision dos 2209 casos. Bol Hosp Viña Del Mar 1985;41:46-50.

3. Bhopal RS, Parkin DW, Gillie RF, Han KH. Pattern of ophthalmologic accidents and emergencies presenting to hospitals. J Epidemiol Community Health 1993;47:382-7

4. Layaun SEED, Schor P, Rodrigues MLV. Perfil da demanda de um serviço de oftalmologia em uma unidade de emergência. Rev Bras Oftalmol 1992;51:171-3.

5. Liu G, Liang KY. Sample size calculations for studies with correlated observations. Biometrics 1997;53:937-47.

6. Edwards RS. Ophthalmic emergencies in a district general hospital casualty department. Br J Ophthalmol 1987;71:938-42.

7. Burton RC. An audit of Worcester Eye Hospital Casualty Department. Massachusetts: USA. Worcester 9-May and June 1989.

8. Jones NP, Hayward JM, Khaw PT, Claoué CM, Elkington AR. Function of an ophthalmic "accident and emergency" department: results of a six month survey. Br Med J 1986;292:188-90.

9. Vernon SA. Analysis of all new cases seen in a busy regional center ophthalmic casualty department during a 24-week period. J R Soc Med 1983;76:279-82.

10. Chiapella AP, Rosenthal AR. One year in an eye casualty clinic. Br J Ophthalmol 1985;69:865-70.

\section{CONGRESSO DA SOCIEDADE BRASILEIRA DE RETINA E VÍTREO}

\section{FÓRUM DO GRUPO LATINO-AMERICANO DE ANGIOGRAFIA OCULAR, FOTOCOAGULAC̣ÃOE CIRURGIA VITREORRETINIANA - GLADAOF}

\section{A 25 DE MAIO DE 2002 \\ GRAN MELIÁ HOTEL - SÃO PAULO - SP}

\section{Informações: Meeting Eventos}

tels.: (11) $5511-3849$ / 0379 / 8263 • fax: (11) $5511-3845$ / 6818

e-mail: info@meetingeventos.com.br • home page: www.meetingeventos.com.br 